\title{
Dimethyl Fumarate Can Enhance the Potential Therapeutic Effects of Epidermal Neural Crest Stem Cells in COVID-19 Patients
}

\author{
Anahid Safari ${ }^{1} \cdot$ Zahra Khodabandeh $^{1} \cdot$ Afshin Borhani-Haghighi ${ }^{2}$ \\ Accepted: 24 November 2020 / Published online: 7 January 2021 \\ (C) Springer Science+Business Media, LLC, part of Springer Nature 2021
}

Dear editor,

We found Salehi et al.'s letter [1] very interesting. They proposed to investigate the potential therapeutic effects of epidermal neural crest stem cells (EPI-NCSCs) in covid-19 disease. There are ongoing trials investigating the efficacy of mesenchymal stem cells (MSCs) in treatment of COVID-19. Salehi et al. believed that EPI-NCSCs might be superior to MSCs due to their high proliferation ability, multi-lineage potential, and lower risk of carcinogenicity. EPI-NCSCs are potent sources of growth factors including fibroblast growth factor (FGF), Transforming growth factor (TGF), Insulin-like growth factor 1 (IGF), Vascular endothelial growth factor (VEGF), Brain derived neurotrophic factors (BDNF), Neurotrophin-3 (NT-3), Nerve growth factor (NGF) and glial cell-derived neurotrophic factor (GDNF). The results of a very recent clinical trial which investigated the therapeutic effects of mesenchymal stem cells (MSCs) in covid-19 patients showed the overexpression of major growth factors in transplanted MSCs [2].

Salehi et al. very briefly described, the beneficial effects of the combination of stem cells and pharmacologic agents in various diseases. This writing is a complementary letter on the potential therapeutic benefits of Dimethyl fumarate (DMF) and EPI-NCSCs combination therapy in covid-19 infection.

Dimethyl fumarate (DMF) and its dynamic metabolite mono methyl fumarate (MMF) have been firstly introduced as a treatment of psoriasis. Its clinical application was then

This article belongs to the Topical Collection: Special Issue on COVID19 Pandemic and Stem Cells

Guest Editor: Mariusz Z. Ratajczak

Afshin Borhani-Haghighi

neuro.ab@gmail.com

1 Stem Cells Technology Research Center, Shiraz University of Medical Sciences, Shiraz, Iran

2 Clinical Neurology Research Center, Shiraz University of Medical Sciences, P.O.Box: 7193635899, Shiraz, Iran extended to cure relapsing remitting Multiple Sclerosis (RRMS). Its anti-inflammatory and anti-oxidants effects, particularly through activating nuclear factor erythroid-derived 2(E2)-related factor (Nrf-2) have been reported [3]. Suppressing nuclear translocation of nuclear factor- $\mathrm{kB}$ (NF- $\kappa B$ ) and enhancing the expression of hydroxyl carboxylic acid receptor (HCAR) were also introduced as the other main mechanisms of action. Regulation of NF- $\mathrm{KB}$ activity was reported to be effective in treating Covid-19 infection [4]. The vast anti-inflammatory effects of HCAR in various organs were also reported [5].

DMF, was recently proposed as a potential therapeutic option for Covid-19 patients. Its potential benefits in reducing alveolar cell injury were explained thoroughly with a focus on its Nrf-2 activating mechanisms of action [6]. Virus entry and replication were also explained to be negatively affected by DMF. Activating secretory leukocyte protease inhibitor (SLPI) as an anti-protease and inhibiting transmembrane protease serine 2 (TMRPSS2) as a protease protein were the main mechanisms introduced. Enhancing the expression of antiviral genes retinoic acid-inducible gene-I (RIG-I) and Interferon beta (IFN- $\beta$ ) were presented as the other potential benefits of DMF therapy in covid-19 patients [6]. DMF and MMF also exert a huge immunomodulatory effects through regulating a wide range of immune cells that determine the host innate and acquired immune response. DMF can effectively change the T- helper cells profile from type 1 to type 2 . Not only B and $\mathrm{T}$ cells but also macrophages, dendritic and natural killer cells are affected [6]. The final result of these vast regulatory effects can be the inhibition of cytokine storm which is known as the main triggered factor for the disease severity in covid-19 patients. The results of a new case series recommended the continuation of DMF therapy in younger MS patients with normal lymphocyte count who become infected with COVID-19 in their course of therapy [7]. Other researchers also confirmed the safety of starting DMF treatment in healthy young or pediatric MS patients [7].

Combination of stem cell therapy with pharmacologic agents have been vastly investigated in a wide range of disease 
models. Our recent investigation demonstrated that DMF effectively induced the over expression of EPI-NCSCs trophic factors profile, particularly those with neurotrophic effects [8]. The enhanced trophic factor profile of MSCs applied in treatment of covid-19 patients [2] can be assumed as a potential therapeutic effect of stem cells in this infection.

According to above-mentioned findings, it can be assumed that DMF-treated EPI-NCSCs may have even more beneficial effects in treatment of SARS-Cov-2 infection than EPINCSCs. Considering the previous reports on the beneficial effects of both EPI-NCSCs [9] and DMF [10] in neurological disorders, DMF- treated EPI-NCSCs may possess the greater advantages in neurological complications of SARS-Cov-2 infection.

\section{References}

1. Salehi, M. S., Pandamooz, S., \& Jurek, B. (2020). Epidermal neural crest stem cells as a perspective for COVID-19 treatment. Stem Cell Reviews and Reports, 1-2. https://doi.org/10.1007/s12015-02010028-3.

2. Leng, Z., Zhu, R., Hou, W., Feng, Y., Yang, Y., Han, Q., \& Zhao, R. C. (2020). Transplantation of ACE2(-) mesenchymal stem cells improves the outcome of patients with COVID-19 pneumonia. Aging and Disease, 11(2), 216-228.

3. Safari, A., Badeli-Sarkala, H., Namavar, M. R., KargarAbarghouei, E., Anssari, N., Izadi, S., \& Borhani-Haghighi, A. (2019). Neuroprotective effect of dimethyl fumarate in stroke:
The role of nuclear factor erythroid 2-related factor 2. Iranian Journal of Neurology, 18(3), 108-113.

4. Elkhodary, M. (2020). Treatment of COVID-19 by controlling the activity of the nuclear factor-Kappa B. CellBio, 09, 109-121.

5. Graff, E. C., Fang, H., Wanders, D., \& Judd, R. L. (2016). Antiinflammatory effects of the hydroxycarboxylic acid receptor 2 . Metabolism: Clinical and Experimental, 65(2), 102-113.

6. Hassan, S. M., Jawad, M. J., Ahjel, S. W., Singh, R. B., Singh, J., Awad, S. M., \& Hadi, N. R. (2020). The Nrf2 activator (DMF) and Covid-19: Is there a possible role? Medical Archives (Sarajevo, Bosnia and Herzegovina), 74(2), 134-138. https://doi.org/10. 5455/medarh.2020.74.134-138.

7. Mantero, V., Abate, L., Basilico, P., Balgera, R., Salmaggi, A., Nourbakhsh, B., \& Cordano, C. (2020). COVID-19 in dimethyl fumarate-treated patients with multiple sclerosis. Journal of Neurology. https://doi.org/10.1007/s00415-020-10015-1.

8. Salehi, M. S., Borhani-Haghighi, A., Pandamooz, S., Safari, A., Dargahi, L., Dianatpour, M., \& Tanideh, N. (2019). Dimethyl fumarate up-regulates expression of major neurotrophic factors in the epidermal neural crest stem cells. Tissue and Cell, 56, 114-120.

9. Salehi, M. S., Pandamooz, S., Safari, A., Jurek, B., Tamadon, A., Namavar, M. R., \& Borhani-Haghighi, A. (2020). Epidermal neural crest stem cell transplantation as a promising therapeutic strategy for ischemic stroke. CNS Neuroscience \& Therapeutics, 26(7), 670-681.

10. Safari, A., Fazeli, M., Namavar, M. R., Tanideh, N., Jafari, P., \& Borhani-Haghighi, A. (2017). Therapeutic effects of oral dimethyl fumarate on stroke induced by middle cerebral artery occlusion: An animal experimental study. Restorative Neurology and Neuroscience, 35(3), 265-274.

Publisher's Note Springer Nature remains neutral with regard to jurisdictional claims in published maps and institutional affiliations. 